\title{
ORIGINAL
}

\section{CARACTERÍSTICAS DE LA MORTALIDAD POR CAUSA TÓXICA EN LA COMUNIDAD AUTÓNOMA VASCA DURANTE EL PERIODO 1986-2001}

\section{Alfonso Apellániz y Ricardo Manzanaro}

Euskal Herriko Unibertsitatea. Facultad de Farmacia. Área de Toxicología. Universidad del País Vasco (EHU-UPV).

\section{RESUMEN}

Fundamento: Las intoxicaciones agudas son una frecuente causa de mortalidad, dando lugar a altas cifras de Años Potenciales de Vida Perdidos. Los objetivos de la investigación son conocer la evolución de la mortalidad por causa tóxica aguda en la Comunidad Autónoma Vasca y su variación en función de diferentes variables.

Método: Se analizan los datos de mortalidad por causa tóxica del Registro de Mortalidad de la Comunidad Autónoma Vasca entre los años 1986 a 2001, en conjunto, en función de diversas variables demográficas y según causa de muerte (CIE-9 y CIE-10). El análisis incluye tasas brutas de mortalidad y tasas estandarizadas por población europea. Se calculan los años potenciales de vida perdidos.

Resultados: Durante el periodo estudiado se registraron 1.207 defunciones por causa tóxica aguda, lo que significa el $045 \%$ del total de fallecimientos, con una tasa bruta de 3,58 muertes por 100.000 habitantes. La media de Años Potenciales de Vida Perdidos fue de $2.226,33$ por año, con una tasa de 1,12 por 1.000 . Un $75,97 \%$ de los fallecidos eran hombres, y la edad media de muerte fue de 40,29 años (36,09 en hombres y 52,64 en mujeres). La etiología accidental fue la más frecuente $(82,19 \%)$, seguida de la intencional $(12,43 \%)$, y la indeterminada $(5,38 \%)$-. El envenenamiento accidental por otros fármacos (E-850 en CIE-9, X44 en CIE-10) fue la primera causa de muerte $(42,30 \%$ y $34,75 \%$ respectivamente).

Conclusiones: A lo largo del periodo estudiado se ha producido un incremento en las muertes por causa tóxica aguda, predominando la etiología accidental por fármacos, con diferencias estadísticamente significativas según sexo y edad.

Palabras clave: Tasa de mortalidad. Envenenamiento. Accidentes. Suicidio. Efectos adversos. Toxicidad.

Correspondencia:

Alfonso Apellániz

Euskal Herriko Unibertsitatea-Universidad del País Vasco

Facultad de Farmacia

Paseo de la Universidad, 7

01006 Vitoria-Gasteiz

\section{ABSTRACT \\ Characteristics of the Poisoning \\ Mortality in the 1986-2001 Period in the Autonomous Basque Community, Spain}

Background: Acute drug-related deaths are frequent, giving rise to high Potential Years of Life Lost figures. The objectives of this research are to ascertain the trend in the acute drug-related death rate in the Autonomous Basque Community and the variance thereof in terms of different variables.

Method: The drug-related death rate data from the Autonomous Basque Community's Death Register for the 1986-2001 period was analyzed overall in terms of different demographic variables and by cause of death (ICD-9 and ICD-10). The analysis includes gross death rates and standardized rates by European population. The potential years of life lost are calculated.

Results: Acute drug-related deaths numbering 1,207 in all were recorded during the period under study, totaling $045 \%$ of all deaths, for a gross rate of 3.58 deaths / 100,000 inhabitants. The mean Potential Years of Life Lost were 2,226.33 /year, for a rate of 1.12 / 1000. A total of $75.97 \%$ of these deaths were males, the average age at time of death having been 40.29 years ( 36.09 for males and 52.64 for females). The etiology most often involved was: accidental $(82.19 \%)$, followed by intentional $(12.43 \%)$ and undetermined (5.38\%)-. Accidental poisoning by other drugs (E-850 in ICD-9, X44 in ICD-10) was the leading cause of death ( $42.30 \%$ and $34.75 \%$ respectively)

Conclusions: There has been an increase in the number of acute drug-related deaths throughout the period studied, the accidental prescribed drug overdose being the main cause, entailing statistically significant differences by sex and age.

Key words: Mortality. Poisoning. Accidents. Suicide. Adverse effects. Toxicity. 


\section{INTRODUCCIÓN}

Las intoxicaciones agudas son aquellas que se manifiestan en las primeras 24 horas de exposición. Presentan una notable incidencia $^{1-4}$, constituyendo una importante causa de mortalidad, ascendiendo al $2 \%$ del total de fallecimientos en España ${ }^{2}$. Afecta especialmente a la población menor de 35 años, siendo la tercera causa de muerte entre los varones de 25 a 34 años y la cuarta entre los de 15 a 24 . Son por ello una de las etiologías que presentan cifras elevadas de Años Potenciales de Vida Perdidos ${ }^{6,7}$.

Según la intencionalidad, se clasifican en intoxicaciones accidentales, intencionadas e indeterminadas. Dentro de las primeras hay una amplia variedad de causas: ambientales, alimentarias, laborales, iatrogénicas o relacionadas con la drogadicción. Entre los 15 y los 24 años las muertes por esta etiología, como las sobredosis, suponen entre un 10 y un $15 \%$ del total ${ }^{8-11}$, y representan el mayor porcentaje atribuible en el incremento de la tasa de mortalidad por todas las causas en este grupo de edad, en el periodo 1983$1990^{12}$. Asimismo las intoxicaciones intencionadas incluyen los homicidios y los suicidios. Una proporción significativa de los suicidios, tanto los intentos consumados como los que no, se llevan a cabo mediante la administración de tóxicos por diversos métodos y vías de entrada ${ }^{1,2,13-16}$.

Otro punto de vista para valorar la trascendencia de este grupo de patologías es la presión asistencial no previsible que ocasiona. Según diversos estudios las intoxicaciones agudas suponen alrededor de un $1 \%$ de las consultas en los servicios de urgen$\operatorname{cia}^{1,14,17-19}$.

En función de estos datos se constata la necesidad de contar con información suficiente y fiable acerca de las intoxicaciones agudas, con el objetivo de establecer estrategias de control y prevención, buscando reducir la incidencia y mortalidad de las mismas.
Para ello hay diferentes métodos de obtención de los datos, como los registros de mortalidad, los sistemas de toxicovigilancia ${ }^{20}, \mathrm{o}$ la elaboración de estudios epidemiológicos en centros sanitarios, tales como servicios de urgencia, áreas de salud, hospitales, etcétera.

Los objetivos de este trabajo son el análisis de la evolución de los datos sobre mortalidad por intoxicación aguda en la Comunidad Autónoma Vasca en los años 1986-2001 en función de diversas variables.

\section{MATERIAL Y MÉTODOS}

Ámbito del estudio: El estudio analiza los datos de mortalidad por causa tóxica aguda en la Comunidad Autónoma Vasca (2.111.078 habitantes a 31 de Diciembre de 2001), así como en cada uno de sus territorios históricos: Bizkaia (1.136.451 habitantes), Gipuzkoa (683.441 habitantes), y Araba (291.186 habitantes), en el intervalo de años comprendido entre 1986 y 2001.

Causas de defunción seleccionadas: Durante el periodo, codificado según CIE-9, las causas seleccionadas fueron: Envenenamiento accidental (E 850-E 869), Exposición a humos o fuegos (E 890-E 891), Efectos adversos de fármacos (E 930-947), Suicidio (E 950-952), Envenenamiento con intención indeterminada (E 980 - E 982). En los años en que la causa de defunción se codificó según CIE-10, las causas seleccionadas, con sus códigos, fueron: Exposición a humos o fuegos (X 01 - X 09), Envenenamiento accidental (X 40 - X 49), Envenenamiento autoinflingido (X 61- X 69), Envenenamiento de intención indeterminada (Y 11 Y 19) y Efectos adversos de fármacos (Y 40 - Y 57).

Fuentes de información: Se han utilizado las estadísticas de mortalidad proporcionadas por el Registro de Mortalidad de la Comunidad Autónoma Vasca (CAV). Las tasas de mortalidad en la población total, y 
para cada uno de los sexos, grupos de edad y causas se calcularon a partir de los datos del Eustat (Euskal Estatistika Erakundea (Instituto Vasco de Estadística) de población estimada de la CAV, en su serie de indicadores demográficos, para los años que comprende el estudio. Como población de referencia se utilizó la europea según los datos de Eurostat. La causa de defunción se codificó de 1986 a 1998 según la Clasificación Internacional de Enfermedades, novena revisión (CIE-9), y de 1999 a 2001 según la CIE-10

Análisis estadístico: Se realizó un análisis general demográfico-geográfico en el que se calculó el número de defunciones por causas tóxicas agudas, así como las tasas brutas de mortalidad durante el periodo mencionado en conjunto y según diversas variables: año, sexo, edad y territorio histórico. Igualmente se obtuvieron las tasas específicas por grupos de edad, agrupados en intervalos de 10 años. Se hallaron las tasas estandarizadas para el total de la población, y para cada uno de los sexos, para cada año y para el total del período estudiad. Estas tasas fueron calculadas por el método directo, utilizando como referencia la población europea. Por último, se calcularon los Años Potenciales de Vida Perdidos (APVP) según la fórmula $\mathrm{APVP}=\Sigma \mathrm{D}_{\mathrm{i}} \mathrm{A}_{\mathrm{i}}$ : Di número de defunciones en el grupo de edad i, Ai diferencia entre 70 años y el punto medio del intervalo de edad i -, así como la tasa de APVP en conjunto y según el año o la causa.

Se llevó a cabo un análisis etiológico dividiendo los datos en tres grandes grupos de envenenamientos (accidental, intencional e indeterminado) y por causa concreta de muerte, según la CIE-9 o la CIE-10. Dentro de cada grupo de envenenamientos y de cada causa de muerte se estratificó por año, sexo, grupo de edad y territorios históricos.

Se valoró la posibilidad de diferencias significativas en la distribución de las frecuencias absolutas de las diversas causas de mortalidad, según las variables contempla- das en el estudio, mediante la prueba de chi2. El nivel de significación elegido fue $\mathrm{p}<0,05$. Todos los análisis se realizaron mediante los programas SPSS y EPI-Info.

\section{RESULTADOS}

Durante el periodo estudiado se registraron 1.207 defunciones por causas tóxicas agudas, lo cual supone una tasa bruta de mortalidad de 3,58 por 100.000 habitantes, y el $0,45 \%$ del total de fallecimientos que tuvieron lugar en dicho periodo.

El año en que se registraron mayor número de fallecimientos fue 1996 con 125 $(10,35 \%$ del total de muertes por esta causa durante el periodo de estudio), y el menor número se registró en 1987 con 23 (1,90\%). Los años en que las intoxicaciones agudas representaron un mayor porcentaje del total de fallecimientos fueron $1996(0,69 \%)$ y $1993(0,62 \%)$, y en los que menor 1987 $(0,15 \%)$ y $1988(0,16 \%)$. La tasa de mortalidad estandarizada, tomando como referencia la población europea fue de 20,72 muertes por 100.000 (tabla 1).

Del total de individuos fallecidos 917 eran hombres $(75,97 \%)$-tasa bruta 5,54 por $100.000-$, y 290 mujeres $(24,03 \%)$ - tasa 1,67. Los años con mayor proporción de hombres entre los fallecidos fueron 1992 $(88,2 \%)$ y $1993(86,4 \%)$ y en los que el porcentaje de mujeres fue más elevado en 1995 $(31,2 \%)$ y $1986(31 \%)(\mathrm{P}<0,01$ en la distribución de sexo de los fallecidos según año).

Por edades el $34,22 \%$ de las muertes se dio en personas de 30 a 39 años, y el 31,23\% entre los 20 a 29. En los hombres el 39,04\% de los fallecimientos aconteció entre los 30 y los 39 años, y el 35,88\% entre los de 20 a 29 años. El 18,97\% de las muertes en mujeres se dio entre los 30 y 39 años, y el 18,28\% entre las de 80 a $89(\mathrm{P}<0,01$ en la proporción de edades según sexo) (tabla 2). Por territorios históricos 676 defunciones (56\%) tuvie- 
Tabla 1

Fallecimientos por intoxicaciones agudas durante el periodo 1986-2001 en la Comunidad Autónoma Vasca por cada año

\begin{tabular}{|l|c|c|c|c|}
\hline Año & $\begin{array}{c}\mathbf{N}^{\mathbf{0}} \text { muertes } \\
-\% \text { muertes en el año - } \\
\text { (\% muertes intox aguda) }\end{array}$ & $\begin{array}{c}\text { Mortalidad } \\
\text { acumulada } \\
\text { \% sobre total) }\end{array}$ & $\begin{array}{c}\text { Tasa bruta } \\
\text { (por 100.000 } \\
\text { habitantes) }\end{array}$ & $\begin{array}{c}\text { Tasa } \\
\text { estandarizada } \\
\text { (por 100.000 } \\
\text { habitantes) }\end{array}$ \\
\hline 1986 & $29-0,19-(2,40)$ & $29(2,40)$ & 1,35 & 3,95 \\
\hline 1987 & $23-0,15-(1,90)$ & $52(4,31)$ & 1,08 & 2,93 \\
\hline 1988 & $25-0,16-(2,07)$ & $77(6,38)$ & 1,17 & 6,07 \\
\hline 1989 & $27-0,17-(2,24)$ & $104(8,62)$ & 1,28 & 6,95 \\
\hline 1990 & $69-0,42-(5,72)$ & $173(14,33)$ & 3,27 & 17,19 \\
\hline 1991 & $97-0,58-(8,04)$ & $270(22,37)$ & 4,60 & 25,34 \\
\hline 1992 & $76-0,46-(6,30)$ & $346(28,67)$ & 3,61 & 21,57 \\
\hline 1993 & $103-0,62-(8,53)$ & $449(37,20)$ & 4,90 & 29,87 \\
\hline 1994 & $90-0,54-(7,46)$ & $539(44,66)$ & 4,28 & 26,71 \\
\hline 1995 & $77-0,44-(6,38)$ & $616(51,04)$ & 3,67 & 22,92 \\
\hline 1996 & $125-0,69-(10,35)$ & $741(61,39)$ & 5,95 & 35,80 \\
\hline 1997 & $96-0,55-(7,95)$ & $837(69,34)$ & 4,56 & 26,90 \\
\hline 1998 & $111-0,61-(9,20)$ & $948(78,54)$ & 5,27 & 26,86 \\
\hline 1999 & $86-0,47-(7,12)$ & $1034(85,66)$ & 4,09 & 25,29 \\
\hline 2000 & $82-0,45-(6,79)$ & $1116(92,45)$ & 3,90 & 24,16 \\
\hline 2001 & $91-0,50-(7,55)$ & 1207 & 4,31 & 23,89 \\
\hline Total & $1207-0,45-$ & 1207 & 3,58 & 20,72 \\
\hline & & & & \\
\hline
\end{tabular}

ron lugar en Bizkaia - tasa bruta 3,67 muertes por 100.000 habitantes -, 413 en Gipuzkoa $(34,21 \%)$-tasa 3,79-, y 118 en Araba $(9,79 \%)$-tasa 2,64-. Los años en que fue mayor la proporción de Bizkaia fueron 2001 $(68,1 \%)$ y $1995(66,2 \%)$, y menor en 1988 $(8 \%)$ y $1989(29,6 \%)(\mathrm{P}<0,01) . \mathrm{La}$ edad media de fallecimiento en Bizkaia fue de 39,06 años; en Guipúzcoa de 41,22 años; y en Araba de 36,59 años.

Según grupos etiológicos el envenenamiento accidental fue la primera causa de mortalidad (992 fallecimientos; 82,19\% del total), seguido del intencional (150 muertes, $12,43 \%)$, y del de intencionalidad indeterminada (65 fallecimientos, 5,38\%) (tabla 3 ).
Según grandes grupos de causas de muerte 21,22, dentro de la categoría «causas externas» (E 800 a E 999; V01 a V89), las intoxicaciones agudas fueron el $8,95 \%$ del total de fallecimientos en la Comunidad Autónoma Vasca en el conjunto de los años del estudio. Los años en que dicha proporción resultó mayor fueron 1996 $(14,70 \%)$ y $1998(12,42 \%)$, y menor 1989 $(1,85 \%)$ y $1988(3,17 \%)$. Dentro del periodo clasificado según la CIE-9, el envenenamiento accidental por «otros fármacos» (E-858) representó el 42,30\%. A continuación se situaron los accidentes por analgésicos (E-850) -17,51\%-, y los suicidios por sustancias sólidas o líquidas (E-950) $8,83 \%$ - (tabla 4). 
Figura 1

Número de fallecimientos por intoxicaciones agudas durante el periodo 1986-2001 en la Comunidad Autónoma y tasa bruta de mortalidad

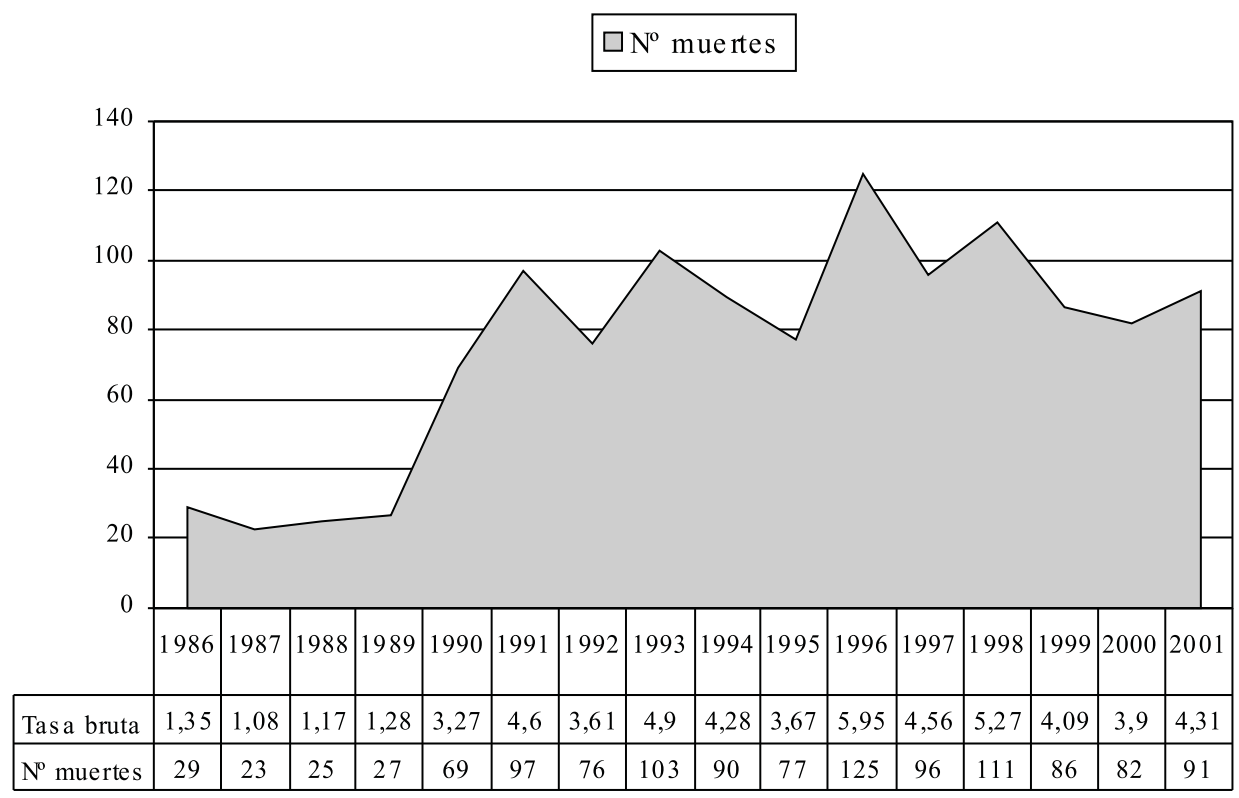

Tabla 2

Distribución por sexo y edad de los fallecidos por causa tóxica aguda durante el periodo de estudio

\begin{tabular}{|c|c|c|c|}
\hline Edad & $\begin{array}{c}\text { Sexo masculino } \\
\text { N (\%) }\end{array}$ & $\begin{array}{c}\text { Sexo femenino } \\
\text { N (\%) }\end{array}$ & $\begin{array}{c}\text { Ambos sexos } \\
\mathbf{N}(\mathbf{\%})\end{array}$ \\
\hline $\mathbf{0 - 9}$ & $3(0,33)$ & $3(1,03)$ & $6(0,50)$ \\
\hline $\mathbf{1 0 - 1 9}$ & $16(1,74)$ & $15(5,17)$ & $31(2,57)$ \\
\hline $\mathbf{2 0 - 2 9}$ & $329(35,88)$ & $48(16,55)$ & $377(31,23)$ \\
\hline $\mathbf{3 0 - 3 9}$ & $358(39,04)$ & $55(18,97)$ & $413(34,22)$ \\
\hline $\mathbf{4 0 - 4 9}$ & $72(7,85)$ & $28(9,65)$ & $100(8,28)$ \\
\hline $\mathbf{5 0 - 5 9}$ & $48(5,23)$ & $12(4,14)$ & $60(4,97)$ \\
\hline $\mathbf{6 0 - 6 9}$ & $33(3,60)$ & $23(7,93)$ & $56(4,64)$ \\
\hline $\mathbf{7 0 - 7 9}$ & $32(3,49)$ & $35(12,07)$ & $67(5,55)$ \\
\hline $\mathbf{8 0 - 8 9}$ & $23(2,51)$ & $53(18,28)$ & $76(6,30)$ \\
\hline $\mathbf{9 0 - 9 9}$ & $3(0,33)$ & $18(6,21)$ & $21(1,74)$ \\
\hline Total & 917 & 290 & 1207 \\
\hline
\end{tabular}


Tabla 3

Distribución de fallecimientos por causa tóxica aguda en función de grupos etiológicos, en conjunto, según sexo y según tramos de edad de fallecimiento

\begin{tabular}{|c|c|c|c|c|}
\hline \multirow{2}{*}{ N (\%) } & \multicolumn{3}{|c|}{ Etiología } & \multirow{2}{*}{ Total } \\
\cline { 2 - 4 } & Accidental & Intencional & Indeterminado & \\
\hline Total & $992(82,19)$ & $150(12,43)$ & $65(5,38)$ & 1207 \\
\hline Masculino & $778(84,8)$ & $97(10,6)$ & $42(4,6)$ & 917 \\
\hline Femenino & $214(73,8)$ & $53(18,3)$ & $23(7,9)$ & 290 \\
\hline$<\mathbf{2 0}$ & $31(83,8)$ & $2(5,4)$ & $4(10,8)$ & 37 \\
\hline $\mathbf{2 0 - 3 9}$ & $698(88,35)$ & $72(9,11)$ & $20(2,53)$ & 790 \\
\hline $\mathbf{4 0 - 5 9}$ & $93(58,12)$ & $46(28,76)$ & $21(13,12)$ & 160 \\
\hline $\mathbf{6 0 - 7 9}$ & $85(69,10)$ & $25(20,32)$ & $13(10,57)$ & 123 \\
\hline$>\mathbf{7 9}$ & $85(87,63)$ & $5(5,15)$ & $7(7,22)$ & 97 \\
\hline
\end{tabular}

Tabla 4

Distribución fallecimientos por intoxicación aguda en función de causa de fallecimiento, según sexo y en total, en las 9 causas de intoxicación más frecuentes, durante el periodo clasificado por CIE-9 (1986-1998)

\begin{tabular}{|c|c|c|c|}
\hline Causa de Fallecimiento & $\begin{array}{c}\text { Hombres } \\
\text { N (\% sobre total) }\end{array}$ & $\begin{array}{c}\text { Mujeres } \\
\text { N (\% sobre total) }\end{array}$ & $\begin{array}{c}\text { Ambos sexos } \\
\text { N (\% sobre total) }\end{array}$ \\
\hline $\begin{array}{c}\text { Envenenamiento accidental por otros } \\
\text { fármacos (E-858) }\end{array}$ & $358(48,97)$ & $43(19,82)$ & $401(42,30)$ \\
\hline $\begin{array}{c}\text { Envenenamiento accidental por analgésicos } \\
\text { (E-850) }\end{array}$ & $146(19,97)$ & $20(9,21)$ & $166(17,51)$ \\
\hline $\begin{array}{c}\text { Suicidio sustancias sólidas y líquidas } \\
\text { (E-950) }\end{array}$ & $44(6,02)$ & $35(16,31)$ & $79(8,33)$ \\
\hline Efectos adversos de fármacos (E930-947) & $23(3,15)$ & $37(17,05)$ & $60(6,33)$ \\
\hline $\begin{array}{c}\text { Envenenamiento accidental otro gas uso } \\
\text { general / CO (E-868) }\end{array}$ & $29(3,97)$ & $21(9,68)$ & $50(5,27)$ \\
\hline $\begin{array}{c}\text { Envenenamiento accidental otras sustancias } \\
\text { sólidas o líquidas (E-866) }\end{array}$ & $32(4,38)$ & $10(4,61)$ & $42(4,43)$ \\
\hline $\begin{array}{c}\text { Suicidio gases y vapores } \\
\text { (E 951-952) }\end{array}$ & $29(3,97)$ & $5(2,30)$ & $34(3,59)$ \\
\hline $\begin{array}{c}\text { Envenenamiento accidental humo incendio } \\
\text { (E 890-891) }\end{array}$ & $14(1,91)$ & $15(6,91)$ & $29(3,06)$ \\
\hline $\begin{array}{c}\text { Envenenamiento accidental por otros gases } \\
\text { (E 980) }\end{array}$ & $15(2,05)$ & $13(5,99)$ & $28(2,95)$ \\
\hline
\end{tabular}


En los años finales del estudio, clasificados según CIE-10, el envenenamiento accidental por «otras drogas, medicamentos, sustancias biológicas» (X 44) representó el $34,75 \%$. El envenenamiento accidental por «narcóticos y psicodislépticos (alucinógenos)» fue la segunda causa más frecuente $11,58 \%$ - y los efectos adversos de fármacos de acción cardiovascular (Y52) la tercera $6,56 \%$ - (tabla 5).

En el grupo de intoxicaciones según la CIE-9, la edad media de fallecimientos fue de 38,54 - 34,49 en hombres, 50,96 en mujeres -. La causa con edad media de muerte más alta fue «efectos adversos de fármaco» -E 930 - E 947-, con 73,8 años, y la más baja «envenenamiento accidental por analgésicos» con 28,3 años- En los casos clasificados en función de CIE-10, la causa de edad media más alta fue efectos adversos de fármacos cardiovasculares con 86,47 años, y la más baja fue envenenamiento accidental por narcóticos y psicodislépticos con 33,43 años (tabla 6).

Durante el periodo de estudio, el número total de años potenciales de vida perdidos (APVP) fue de 37.773, con una tasa de APVP de 1,12 por 1.000 , y con una media anual de 2.226,33 años. Los años con mayor cifra de APVP fueron 1996 (4064, tasa 1,93 por 1.000$)$ y $1993(3719$, tasa 1,77$)$, y los de menor $1987(417$, tasa 0,20) y 1986 (527, tasa 0,24$)$.

Según grupos etiológicos, las intoxicaciones accidentales supusieron un total de 32.200 APVP (tasa 0,95 por 1.000), las intencionadas 4071 APVP (tasa 0,12 por 1.000) y las indeterminadas 1502 APVP (tasa 0,04 por 1.000 )

Las etiologías que mayor número de APVP ocasionaron fueron envenenamiento accidental por otros fármacos $(13.460$

Tabla 5

Distribución fallecimientos por intoxicación aguda en función de causa de fallecimiento, según sexo y en total, en las 9 causas de intoxicación más frecuentes, durante el periodo clasificado por CIE-10 (1999-2001)

\begin{tabular}{|c|c|c|c|}
\hline Causa de Fallecimiento & $\begin{array}{c}\text { Hombres } \\
\text { N (\% sobre } \\
\text { total) }\end{array}$ & $\begin{array}{c}\text { Mujeres } \\
\text { N (\% sobre total) }\end{array}$ & $\begin{array}{c}\text { Ambos sexos } \\
\text { N (\% sobre total) }\end{array}$ \\
\hline $\begin{array}{c}\text { Exposición otras drogas, medicamentos, sust } \\
\text { biológicas (X 44) }\end{array}$ & $\begin{array}{c}77(41,40) \\
\text { Exposición narcóticos y psicodislépticos (X 42) }\end{array}$ & $26(13,98)$ & $4(34,75)$ \\
\hline $\begin{array}{c}\text { Efectos adversos otros agentes acción cardiovascular } \\
\text { (Y 52) }\end{array}$ & $3(1,61)$ & $14(19,18)$ & $17(6,56)$ \\
\hline $\begin{array}{c}\text { Efectos adversos agentes que afectan a constituyentes } \\
\text { sangre (Y 44) }\end{array}$ & $8(4,30)$ & $5(6,85)$ & $13(5,02)$ \\
\hline $\begin{array}{c}\text { Exposición humos fuegos en vivienda (X 09) } \\
\text { Envenenamiento autoinflingido otras drogas } \\
\text { medicamentos (X 64) }\end{array}$ & $8(4,30)$ & $3(4,11)$ & $11(4,25)$ \\
\hline $\begin{array}{c}\text { Envenenamiento autoinflingido por exposición gases } \\
\text { vapores (X 67) }\end{array}$ & $9(4,84)$ & $5(6,85)$ & $10(3,86)$ \\
\hline $\begin{array}{c}\text { Envenenamiento accidental exposición gases y } \\
\text { vapores (X 47) }\end{array}$ & $5(2,69)$ & $3(1,37)$ & $8(3,86)$ \\
\hline $\begin{array}{c}\text { Envenenamiento autoinflingido exposición drogas } \\
\text { antiepilépticos (X61) }\end{array}$ & $3(1,61)$ & $5(6,85)$ & $8(3,09)$ \\
\hline
\end{tabular}


Tabla 6

Edad media de los fallecidos por intoxicación aguda en función de la causa de fallecimiento, en las 8 causas más frecuentes en cada uno de los periodos clasificados según CIE-9 y CIE - 10

\begin{tabular}{|c|c|c|c|}
\hline $\begin{array}{c}\text { Causa } \\
\text { CIE - } 9(1986-1998)\end{array}$ & $\begin{array}{c}\text { Edad media }+/- \text { DS } \\
(\mathbf{N})\end{array}$ & $\begin{array}{c}\text { Causa } \\
\text { CIE-10 (1999-2001) }\end{array}$ & $\begin{array}{l}\text { Edad media }+/-D S \\
(\mathbf{N})\end{array}$ \\
\hline $\begin{array}{c}\text { E-858 } \\
\text { E. acc otros fármacos }\end{array}$ & $\begin{array}{c}39,26+/-9,85 \\
(401)\end{array}$ & $\begin{array}{c}\mathrm{X}-44 \\
\text { Env. acc otras drogas }\end{array}$ & $\begin{array}{c}34,43+/-7,11 \\
(90)\end{array}$ \\
\hline $\begin{array}{c}\text { E-850 } \\
\text { E. acc analgésicos }\end{array}$ & $\begin{array}{l}28,30+/-5,01 \\
(166)\end{array}$ & $\begin{array}{c}\text { X } 42 \\
\text { E. acc narcóticos }\end{array}$ & $\begin{array}{c}33,43+/-4,61 \\
(30)\end{array}$ \\
\hline $\begin{array}{c}\text { E-950 } \\
\text { Suicidio sust sol liq. }\end{array}$ & $\begin{array}{c}43,97+/-18,18 \\
(79)\end{array}$ & $\begin{array}{c}\text { Y-52 } \\
\text { Efectos adversos Carpio }\end{array}$ & $\begin{array}{c}86,47+/-7,67 \\
(17)\end{array}$ \\
\hline $\begin{array}{c}\text { E-930-947 } \\
\text { Effect. Adv. Fármacos }\end{array}$ & $\begin{array}{c}73,80+/-18,78 \\
(60)\end{array}$ & $\begin{array}{c}\text { Y } 44 \\
\text { Efect. adv. agentes sangre }\end{array}$ & $\begin{array}{c}70,15+/-9,70 \\
(13)\end{array}$ \\
\hline $\begin{array}{c}\mathrm{E}-868 \\
\text { E. acc otro gas uso general / CO }\end{array}$ & $\begin{array}{l}51+/-28,26 \\
\quad(50)\end{array}$ & $\begin{array}{c}\text { X09 } \\
\text { Exposic. otros humos }\end{array}$ & $\begin{array}{c}55+/-22,27 \\
(11)\end{array}$ \\
\hline $\begin{array}{c}E-866 \\
\text { E. acc otras sust sol }- \text { liq }\end{array}$ & $\begin{array}{c}33,98+/-17,82 \\
(42)\end{array}$ & $\begin{array}{l}\text { X } 64 \\
\text { Autoinfling. otras drogas }\end{array}$ & $\begin{array}{c}48,9+/-15,57 \\
(42)\end{array}$ \\
\hline $\begin{array}{c}E-951-952 \\
\text { Suicidio gases vapores }\end{array}$ & $\begin{array}{c}42,22+/-15,87 \\
(34)\end{array}$ & $\begin{array}{c}\text { X } 67 \\
\text { Autoinfling. otros gases }\end{array}$ & $\begin{array}{c}38,2+/-13,97 \\
(10)\end{array}$ \\
\hline $\begin{array}{c}\text { E } 890-891 \\
\text { E. acc humo incendio }\end{array}$ & $\begin{array}{c}57,57+/-24,10 \\
(29)\end{array}$ & $\begin{array}{c}\text { X } 47 \\
\text { Env. acc otros gases }\end{array}$ & $\begin{array}{c}47,38+/-24,88 \\
(8)\end{array}$ \\
\hline
\end{tabular}

APVP, tasa 0,40 por 1.000$)$, por analgésicos (6.574 APVP, tasa 0,19 por 1.000 ) y por otras sustancias sólidas o líquidas (1555 APVP, tasa 0,05 por 1.000$)$.

\section{DISCUSIÓN}

En el análisis de los resultados se observa un incremento de la incidencia de las intoxicaciones agudas a partir de 1990, y de la proporción sobre el total de fallecimientos.

Por sexos predomina en hombres, no observándose una tendencia clara en los datos en el transcurso de los años. Al ser la edad media de fallecimiento es de 40,29 años, y producirse el $65,45 \%$ de las muertes en personas de 20 a 39 años esta etiología ocasiona un elevado nivel de Años Potenciales de Vida Perdidos. Según sexo, en todos loa años del estudio es más elevada la edad media en mujeres. Al comparar los datos de la edad media de fallecimiento por causas tóxicas agudas con los correspondientes por causas externas y por todas las etiologías en la Comunidad Autónoma Vasca resulta que la primera es inferior a la global pero superior a la media de muertes por causas externas.

Por causas, hay un predominio de la etiología accidental sobre la intencional, o la indeterminada. Se observa una mayor proporción de los suicidios en mujeres, así como de los de causa indeterminada. Igualmente, dentro de los accidentes, es mayor la proporción de reacciones adversas a fármacos en mujeres.

Aunque en todos los tramos de edad la causa de defunción principal es la accidental, se incrementan significativamente la intencional y la indeterminada entre los $40 \mathrm{y}$ los 79 años. Por causas concretas de fallecimiento, en edades más jóvenes predominan 
los envenenamientos accidentales por drogas, mientras que en los de más edad son los efectos adversos de fármacos, con una proporción creciente del suicidio de los 30 a los 60 años.

Al comparar los datos registrados en cada una de las capitales, con los del resto de la provincia, únicamente se obtuvieron algunas diferencias en Álava respecto al envenenamiento accidental por analgésicos, otros fármacos (mayor proporción en la capital) y por otros gases y $\mathrm{CO}$ (más porcentaje en la provincia) ( $p<0,01$ en los dos primeros y $p=0,02$ en el último).

Del análisis de otros estudios sobre mortalidad por causa tóxica aguda, destaca que en la mayoría de los mismos la etiología más frecuente es la intencional (en algunos de ellos se incluye en dicha categoría los causados por sobredosis, además del suicidio $)^{1,4,15,17}$, y son escasos en los que, al igual que el presente estudio, el envenenamiento accidental supone un mayor porcentaje que el suicidio ${ }^{18}$. Se observa que la intoxicación no intencional es más frecuente en hombres, y el suicidio entre las mujeres ${ }^{4,15}$. Asimismo, las benzodiacepinas son el fármaco más frecuente en los suicidios ${ }^{15}$. La letalidad de la intoxicación aguda oscila entre 0,23 y $6 \%$ 1,5,14,19-21.

Examinando estudios sobre intoxicaciones agudas en general se observa que éstas suponen entre un $0,18 \%$ y un $0,69 \%$ del total de consultas atendidas en servicios de urgencias $^{11-13}$. La edad media de los intoxicados está en torno a los 35 años, oscilando desde $\operatorname{los} 27$ a los 3911-13,21,22. Es mayor la proporción de estudios en los que el sexo más frecuente entre los intoxicados es el masculino ${ }^{12,13,16,17,23}$, frente a aquéllos de predominio femenino ${ }^{11,22}$

Con respecto a la intencionalidad no hay unos resultados homogéneos en los estudios revisados, con un mayor número de casos debido a accidentes en algunos ${ }^{1,13,23}$, y un predominio de la etiología voluntaria (suicidio y sobredosis) en otros ${ }^{11,12,14,20,24,25}$. Dentro de las intoxicaciones accidentales son más frecuentes entre hombres y jóvenes, así como en el ámbito doméstico. En éste los tóxicos más habituales son los productos caústicos ${ }^{1,12,26,27}$. Entre los voluntarios son un poco más frecuentes, con alguna excepción, los intentos de suicidio frente a sobredosis de alcohol o drogas ${ }^{11-13,20}$. Dentro de los ocasionados por agentes farmacológicos predominan las benzodiacepinas y con bastante menor porcentaje los antidepresivos tricíclicos ${ }^{11-15,21,22,28}$. Son frecuentes en las intoxicaciones medicamentosas voluntarias las asociaciones medicamentos-drogas ${ }^{29,30}$. La intencionalidad autolítica es más frecuente en mujeres y mayor edad y, por el contrario, las intoxicaciones por abuso de alcohol y drogas se dan más en jóvenes y varones $^{3,15,19,24,25}$. Según un estudio elaborado en Inglaterra y Gales analizando los últimos 35 años, los suicidios por envenenamiento han reducido su proporción frente a otros métodos ${ }^{31}$ En el caso de la intoxicación por monóxido de carbono predomina el ámbito doméstico, y los causados por deficiente combustión de butano son los más frecuentes $(31 \%)^{32}$.

Otro grupo de causas, dentro de la etiología accidental es la de la de los efectos adversos de fármacos, los cuales presentan una notable incidencia en las edades avanzadas. Los fármacos implicados más frecuentes son en particular la aspirina, de forma más general los cardiovasculares y los diuréticos. La mortalidad de las intoxicaciones agudas por reacciones adversas está entre el 0,15 y el $0,32 \%^{33,34}$. Un $27,6 \%$ de las intoxicaciones por esta causa son fácilmente prevenibles ${ }^{35}$.

Por último, hay diversos estudios que reflejan la disparidad de los datos de mortalidad, según diversas variables tales como si se trata de un registro de mortalidad, o un sistema de vigilancia epidemiológica ${ }^{36}$, el sistema de registro de datos ${ }^{37} \mathrm{o}$, dentro de un mismo sistema, según la persona que cum- 
plimenta el documento o el momento en que se realiza ${ }^{38}$.

\section{AGRADECIMIENTOS}

Los autores agradecen la colaboración del Registro de Mortalidad de la Dirección de Planificación y Ordenación Sanitaria del Departamento de Sanidad del Gobierno Vasco.

\section{BIBLIOGRAFÍA}

1. Ferrer A, Nogué S, Vargas F, Castillo O. Toxicovigilancia: una herramienta útil en salud pública. Med Clin (Barc), 2000; 115: 236-8.

2. Ruiz Ramos M, Nieto García A. Evolución de la mortalidad por accidentes infantiles y su distribución geográfica en España (1975-1994) Rev Esp Salud Publica, 2001; 75: 433-42.

3. Office of Statistics and Programming. National Center for Injury Prevention and Control CDC. National Estimates of Nonfatal Injuries Treated in Hospitals Departments. United States, 2000. MMWR 2001; 50: 340-6.

4. Llacer A, Fernandez-Cuenca R, Martínez Aragón MV. Mortalidad en España en 1998: evolución en la década 1989-1998. I. Mortalidad general. Principales causas de muerte y años potenciales de vida perdidos. Bol Epidemiol Sem 2001; 9: 241-4.

5. Hauzaaoui N, Whitten P. Causes of death among young people aged 15 to 241994 / 1997. Statistics in focus $2001 ; 3: 1-7$.

6. Montellá N, Borrel C, Brugal MT., Plasencia A. Evolución de la mortalidad de los jóvenes de la ciudad de Barcelona 1983-1995. Med Clin (Barc) 1997; 108: 241-7.

7. Borrel, Pasarín MI, Cirera E, Klutke P, Pipitone E, Plasencia A. Trends in young adult mortality in three european cities: Barcelona, Bologne and Munich, 1986-1995. J Epidemiol Community Health 2001; 55: 577-82.

8. Sánchez J, Rodriguez B, de la Fuente L, Barrio G, Vicente J, Roca J, Royuela L. Opiates or cocaine: mortality from acute reactions in six major Spanish cities. State Information System on Drug Abuse (SETT) Working Group. J. Epidemiol Community Health 1995; 49: 54-60.
9. Torralba L, Brugal MT, Villalbí JR, Tortosa MT, Toribio A, Valverde JL. Mortality due to acute adverse drug reactions: opiates and cocaine in Barcelona 1989-1993. Adicction 1996; 91: 419-26.

10. Fuente de la L, Barrio G, Vicente J, Bravo MJ, Santacreu J. The impact of drug-related deaths on mortality among young adults in Madrid. Am J Pub Health 1995; 85: 102-5.

11. Riquelme Rodríguez A, Burill-Putze G, Jimenez Sossa A. Hardisson de la Torre A. Epidemiología global de la intoxicación aguda en un área de salud. Aten Primaria 2001; 28: 506-7.

12. Caballero Valdés PJ, Dorado Rubio S, Brusint Olivares B, Jerez Basurco B, Medina Sampedro M. Vigilancia epidemiológica de la intoxicación aguda 1997 (estudio de 1.140 casos del área sur de la Comunidad de Madrid). Rev Clin Esp 1999; 199: 424-30.

13. García Bajo A, Santos Pérez ME, Sanz Ortega F, Zapico Alvarez N, Thomson Okatsu K, García Pérez A, Borrás Beats R. Estudio epidemiológico sobre intoxicaciones agudas y dotación de botiquines de antídotos. An Med Interna (Madrid), 1999; 16: $285-9$.

14. Dorado Pombo S, Martín Fernández J, Sabugal Rodelgo G, Caballero Valdés PJ. Epidemiología de la intoxicación aguda: estudio de 613 casos en la Comunidad de Madrid en 1994. Rev Clin Esp 1996; 196: 150-6.

15. Río del Muñoz PA, Cabrera Benet R. Intentos de suicidio por fármacos en España (1991-1992). Rev Esp Med Legal 1997; XXI: 25-32.

16. Canovas A, Oliva de la S, Mencías E. Seguimiento de las evoluciones de intoxicaciones agudas consultadas por centros hospitalarios al servicio de información toxicológica. Rev Toxicol 2001; 18 : 182.

17. Lovitz TL, Klein-Scwartz W, White S, Cobaugh DJ, Youniss J, Omolaer JC, Drab JC, Drab A, Benson BE. 2000 Annual Report of the American Poison Control Centers. Toxic Exposure Surveillance System. Am J Emergency Medicine 2001; 19: 33747.

18. Dirección General de Salud Pública y Dirección de Economía y Estadísticas. Estadísticas básicas de mortalidad en la región de Murcia de 1998. Consejería de Sanidad y Consumo. Consejería de Economía y Hacienda. Murcia; 2000.

19. Jacobsen D, Frederichsen PS, Knutsen KM, Sorum Y, Tabeth Y, Odegaard OR. A prospective study of 
1212 cases of acute poisoning: general epidemiology. Hum Toxicol 1984; 3: 93-100.

20. Cobo Valle M, Martí Lloret JB, Miralles Gisbert S, Martí Criquion JL. Etiology of intoxication: a study of 557 cases. Eur J Epidemiol 1999; 361-7.

21. Hermida I, Fernández P, Ferrer A, Bermejo A, Tabernero MT. Estudio de 999 intoxicaciones agudas atendidas en un hospital. Rev Toxicol 2000; 17: $70-4$.

22. Guevara Serrano J, Torres Baudia F, Ortega García P, Fernández Villalba E, Carmona Ibáñez G. Estudio de las autoagresiones medicamentosas atendidas en urgencias en un hospital general durante un periodo de seis años. Farm Hosp 2000; 24: 248-52.

23. Repetto MR. Epidemiology of poisoning due to pharmaceutical products. Poison Control Center, Sevilla, Spain. Eur J Epidemiol 1997; 13: 353-6.

24. Dorado Pombo SS. Alvarez Nido R, Caballero Valdés PJ, Medina-Asensio J, Casanova García, Granado Garrido JA. Epidemiología de intoxicación aguda: estudio de 851 casos detectados en el sur de la Comunidad d Madrid en 1990. Rev Clin Esp 1992: 191: 131-6.

25. Caballero Valles PJ, Medina Sanpedro M, Brusint Olivares B, Dorado Pombo S, Jerez Basurco B. Vigilancia epidemiológica de la intoxicación aguda en el Área Sur de la Comunidad de Madrid. Estudio VEIA 2000. An Med Interna 2001; 21: 62-8.

26. Laín M, Manteigna M, Morales J, Julián A, Vélez C, Lael P, Sentenac G, Lázaro S. Intoxicaciones agudas atendidas en un servicio de urgencias. Rev Toxicol 2001; 18: 187.

27. Rayo R, Ruiz E, Ferrer A, Rivas M. Intoxicaciones por productos químicos atendidas en urgencias . Rev Toxicol 2001; 18: 187.

28. Pérez Gómez JM, Belzuncegui Otano T. Intoxicaciones medicamentosas agudas voluntarias - IMAV - en el área sanitaria Navarra I, durante 1989. Estudio epidemiológico descriptivo. Rev San Hig Pública 1990; 65: 401-14.

29. Hermida I, Férnandez P, Ferrer A, Bermejo AM, Tabernero MJ. Perfil psicosocial de pacientes ingresados por intoxicación aguda voluntaria. Rev Toxicol 2003; 20: 35-7.

30. Oliver P, Keen J. Concomitant drugs of misuse and drug using behaviours associated with fatal opiaterelated poisoning in Sheffield, UK, 1997-2000. Adicction 2003; 98: 191-7.

31. Flanagan RJ, Rooney C. Recording acute poisoning deaths. Forensic Sci Int 2002; 128: 3-19.

32. Dueñas-Laita A, Ruiz Mambrilla M, Gandía F, Cerda R, Martín-Escudero JC, Pérez Castrillón JL, Diaz G. Epidemiology of acute carbon monoxide poisoning in a spanish region. $\mathrm{J}$ Toxicol Clin Toxicol 2001; 39: 53-7.

33. Pirmohamed M, James S, Meakin S, Green C, Scott AK, Walley TJ, Farrar K, Park K, Breckenridge AM. Adverse drug reactions as cause of admission to hospital: prospective analysis of 18820 patients. BMJ 2004; 329: 15-9.

34. Lazarou J, Pomeranz BH, Corey PN. Incidence of adverse drug reactions in hospitalized patients: a meta-analysis of prospective studies. JAMA 1998; 279: 1200-05.

35. Gurwitz JH, Field TS, Harrold LR, Rothschild J, Debellis K, Seger AC, Cadoret C, Fish LS, Garber L, Kelleher M, Bates DW. Incidence and preventability of adverse drugs events among older persons in the ambulatory setting. JAMA 2003; 289: 115456.

36. Hoppe-Roberts JM, Lloyd LM, Chyka PA. Poisoning mortality in the United States: comparison of National Mortality statistics and poison control centers report. An Emerg Med 2000; 35: 4408.

37. Brugal MT, Barrio G, Regidor E, Mestres M, Cayla JA, Fuente de la L. Discrepancias en el número de muertes por reacción aguda a sustancias psicoactivas registradas en España. Gac Sanit 1999; 13: 79-81.

38. Arán Barrés M, Pérez G, Rosell J, Molina P. Exactitud de las estadísticas y mortalidad por causas externas y naturales con intervención médicolegal en Cataluña, 1996. Gac Sanit 2000; 14: 35662. 
